\title{
May the FTO gene affect visceral obesity? Study of variant rs9930506 SNP among the MONW in a Polish population.
}

Katarzyna Kolačkov ${ }^{1}$, Łukasz Łaczmański ${ }^{1}$, Małgorzata Tupikowska², Aleksandra Zdrojowy-Wełna ${ }^{1}$, Grażyna Bednarek-Tupikowska ${ }^{1}$

Department and Clinic of Endocrinology, Diabetology and Isotope Therapy, Wroclaw Medical University

2) Department and Clinic of Dermatology, Venereology and Allergology, Wroclaw Medical University

\section{Introduction}

Genetic factors play a major role in obesity. Numerous studies have confirmed associations of the SNP rs9930506 of FTO gene with increased BMI, hip circumference and total body weight. The metabolic consequences of obesity depend, to a large degree, on body fat distribution.

MONW individuals (metabolically obese normal-weight) are characterized by visceral obesity and display metabolic properties that may predispose them to developing metabolic syndrome. Because of normal BMI and young age, the MONW individuals are often overlooked in preventive examinations on the pathogenesis of obesity and its metabolically consequences.

\section{FTO gene}

The FTO is a large gene, that is located on the chromosome 16 at position $16 \mathrm{q} 12.2$, consists of 9 exons and spanning more than $400 \mathrm{~kb}$. FTO gene encodes a 2-oxoglutaratedependent nucleic acid demethylase-enzyme occuring many tissues, particularly hypothalamus, which is responsible for certain metabolic processes, control of food intake and energy expenditure.
Aim of research

The aim of this research is to identify and examine the association of the FTO gene polymorphism on amount and distribution of body fat as well as its correlation with metabolic parameters in a large study group of over 800 young, healthy Polish men and women with an isolated group of MONW individuals.

\section{Materials and methods}

The FTO rs 9930506 SNP was genotyped in 824 young (20 to 40 years of age) subjects, randomly selected from tree different regions of Poland. Identification of FTO gene polymorphism was performed using a minisequencing method.

We analyzed the influence of rs9930506 of FTO gene on the body fat distribution and correlated metabolic parameters in two groups: metabolically obese-normal weight (MONW) and normal weight, classified with consideration to HOMA value (1.69).

All subjects had previously undergone physical examinations, anthropometric measurements (BMI, WHR), densitometry (DXA, dual X-ray absorptiometry) and biochemical measurements (triglycerides, total cholesterol, HDL, LDL, glucose, and insulin).

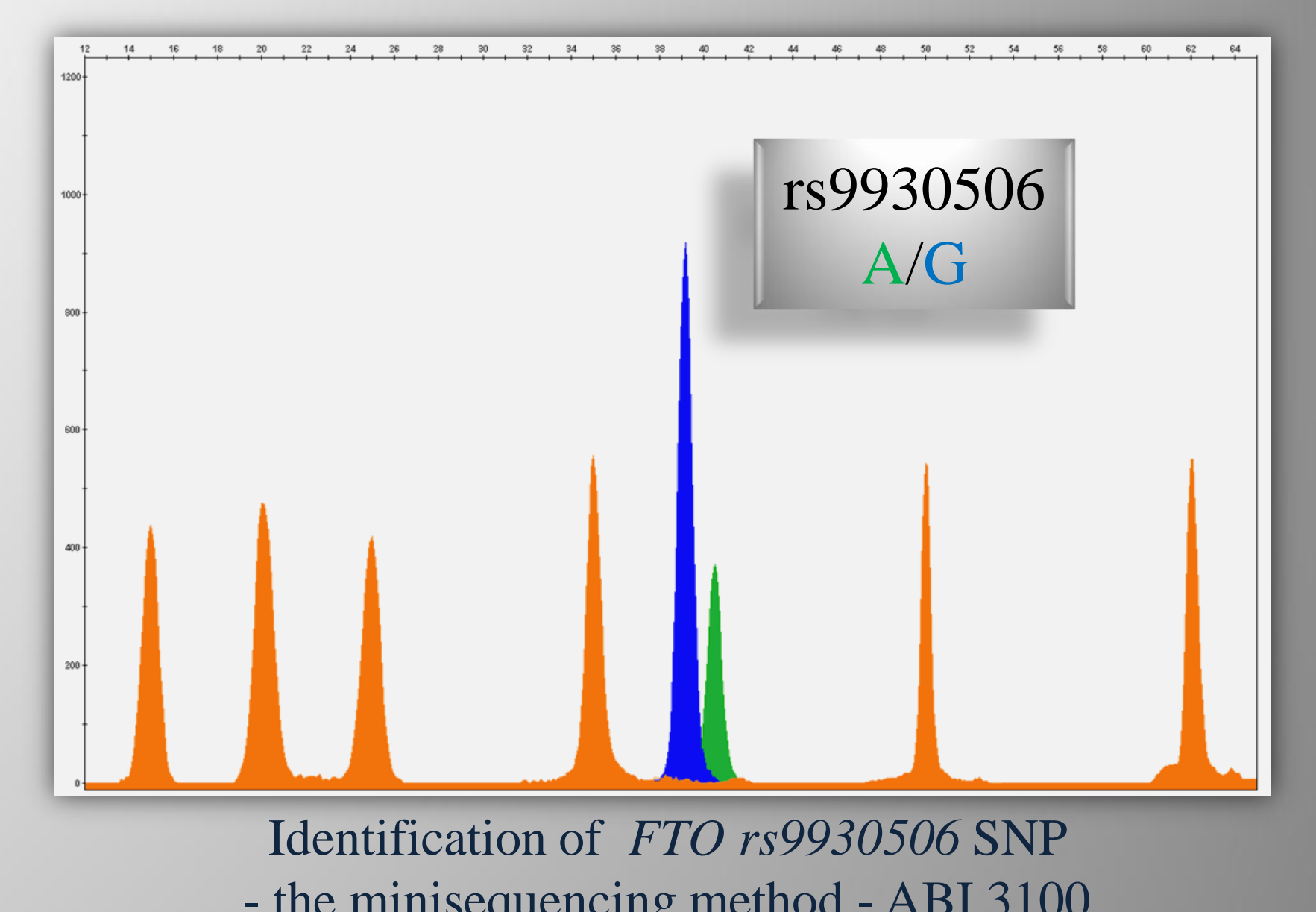

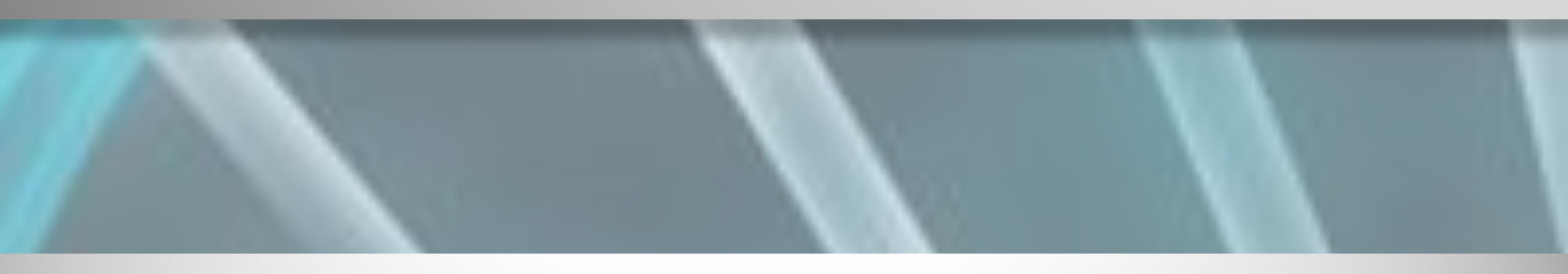

Results

The prevalence of genotypes of the FTO gene was consistent with the Hardy-Weinberg equilibrium in the studied group of men $\left(c h i^{2}=0.05, p=0.8289\right)$ and women $\left(c h i^{2}=0.20\right.$, $p=0.6573$ ). There were no statistical differences in the prevalence of genotypes in MONW and normal weight groups between the two groups.

In the group of normal weight men, in homozygous carriers of the risk allele (GG), higher waist circumference was noted $(p=0.0449)$. In the group of MONW men, the carriers of the risk allele had increased hip circumference $(p=0.0356)$ and T.FAT $(p=0.0350)$.

\section{Conclusion}

Our study shows association with increased waist and hip circumference as well as total fat in homozygous carriers of the risk allele in men. However despite of the FTO rs9930506 SNP association with particular parameters in examined groups, the results did not confirm the impact of this polymorphism on visceral obesity in MONW individuals.

We suppose that the mechanism of accumulation of excessive amounts of visceral adipose tissue in MONW individuals does not depend on the examined gene polymorphism. Further studies are required.
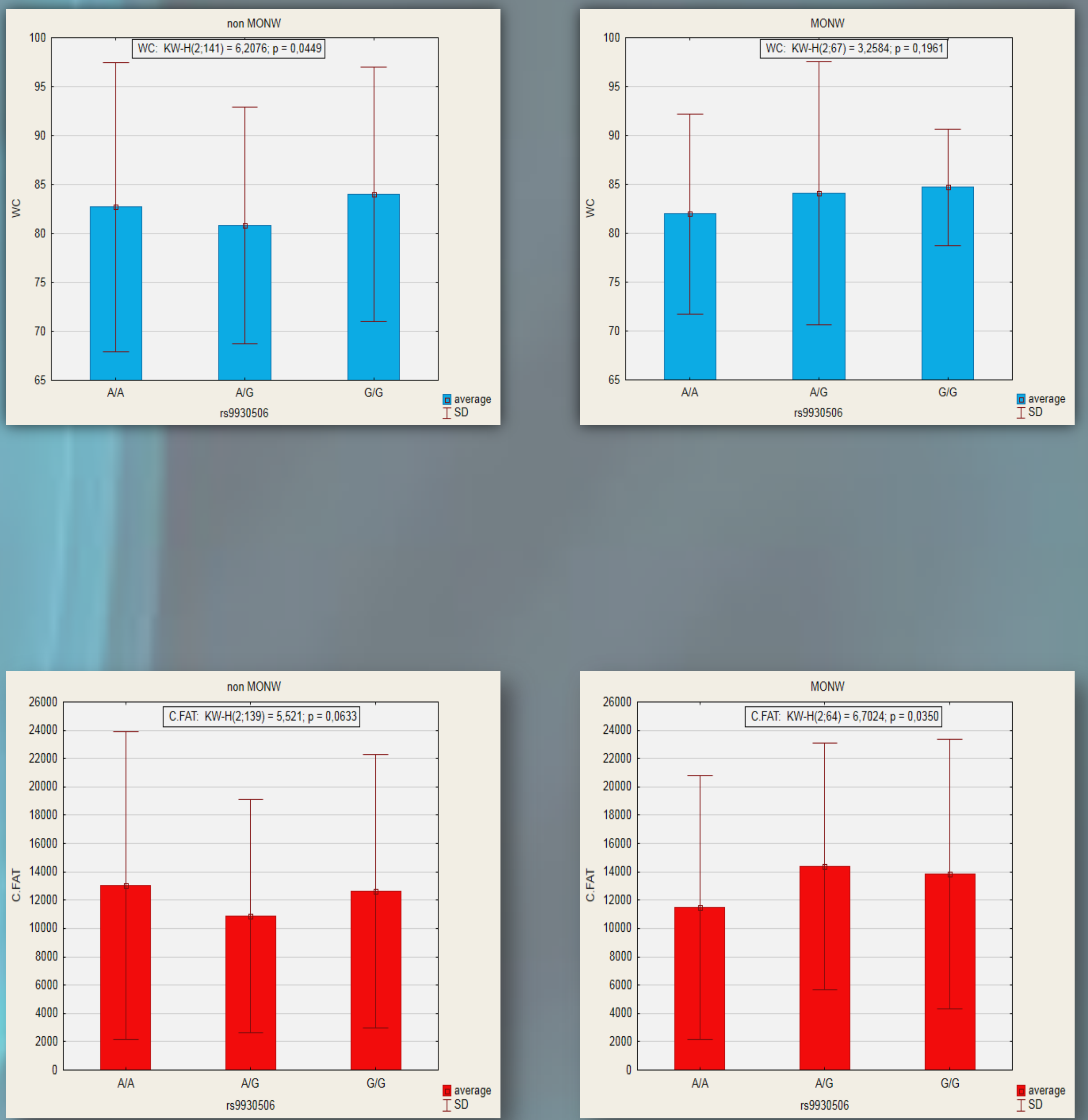